\title{
Specially Coupled Dark Energy in the Oscillating FRW Cosmology
}

\author{
A.V. Timoshkin*
}

Tomsk State Pedagogical University, Tomsk, Russia

\begin{abstract}
We consider a four-dimensional flat-space Friedman universe, which is filled with two interacting ideal fluids (the coupling of dark energy with dark matter of special form). The gravitational equations of motion are solved. It is shown that in some cases there appears a periodic universe with finite-time cosmological singularities and also the universe becomes static in the remote future.
\end{abstract}

\section{INTRODUCTION}

In the present work we investigate a phenomenological model of the coupling of dark energy with dark matter of special form (for a review, see [1]), where there is a dark energy with a non-linear inhomogeneous equation of state and a dark matter with a linear homogeneous equation of state (for a review of similar models in modified gravity, see reference [2]). There is a lot of interest in the study of a nature of the coupling of dark matter with dark energy responsible for the acceleration of cosmic expansion. Among the different possible models that have been considered in the literature there exists a model in which a dark energy and a dark matter are described by the ideal fluid with an unusual equation of state. Very general dark-fluid models with an inhomogeneous equation of state were introduced in the papers [3-5], (see also the examples in [6]). The ideal fluid with a time-dependent non-linear inhomogeneous equation of state has been considered in the papers [7, 8]. Some examples of such kind of equations can be found in papers [9, 10]. It is known that the time-dependence of the parameters in the equation of state of ideal fluid can lead to a transition from the non-phantom era to phantom one in the evolution of the universe (see $[11,12])$. The cases where the parameters $w$ and $\Lambda$ in the equations of state are stationary or they depend linearly on time are also described. Under a corresponding choice of the interaction between a dark energy and a dark matter the expressions for a scale factor and its first and second derivatives are obtained. The values of the parameters where the universe expands with acceleration (quintessence) and with deceleration are found. It is shown that the interaction between a dark energy and a dark matter may lead to a periodical appearance of the cosmological singularities $[1,13]$. Note that time-dependent equation of state fluid may serve as classical analog of string landscape [14] or of modified gravity [15].

*Address correspondence to this author at the Tomsk State Pedagogical University, Tomsk, Russia; E-mail: TimoshkinAV@tspu.edu.ru

\section{THE INFLUENCE OF THE INTERACTION BETWEEN A DARK ENERGY AND A DARK MAT- TER ON THE EVOLUTION OF THE UNIVERSE}

Let us consider a universe filled with two interacting ideal fluids: dark energy and dark matter. In a spatially flat Friedman-Robertson-Walker metric with a scale factor $a(t)$ the background equations are given by [1]:

$$
\left\{\begin{array}{l}
\dot{\rho}+3 H(p+\rho)=-Q \\
\dot{\rho}_{m}+3 H\left(p_{m}+\rho_{m}\right)=Q \\
\dot{H}=-\frac{k^{2}}{2}\left(p+\rho+p_{m}+\rho_{m}\right)
\end{array}\right.
$$

where $H \equiv \frac{\dot{a}}{a}$ is the Hubble rate, and $k^{2}=8 \pi G$, with the Newton's gravitational constant $G ; p, \rho$ and $p_{m}, \rho_{m}$ are the pressure and the energy density of a dark energy and a dark matter correspondingly; $Q$ is the interaction term between a dark energy and a dark matter. Here a dot denotes the derivative with respect to cosmic time $t$.

The Friedman's equation for the Hubble rate is given by [1]:

$$
H^{2}=\frac{k^{2}}{3}\left(\rho+\rho_{m}\right)
$$

First of all we will investigate the stationary case where the parameters $w_{1}, w_{2}$ in the equations of state and the parameter $\Lambda$ don't depend on time:

$$
\begin{aligned}
& p_{m}=w_{1} \cdot \rho_{m} \\
& p=w_{2} \cdot \rho+\Lambda+a_{1} \cdot H
\end{aligned},
$$

where $a_{1}$ is a some constant.

Taking into account equations (1) - (3) we obtain a gravitational equation of motion for the system "energymatter": 


$$
\dot{\bar{\rho}}+\sqrt{3} k \sqrt{\bar{\rho}}\left[(1+w) \bar{\rho}+\Lambda+a_{1} H^{2}\right]=0,
$$

where $\bar{\rho}=\rho+\rho_{m}, w_{1}=w_{2}=w$.

The solution of the equation (4) looks like:

$$
\bar{\rho}=\frac{3 \Lambda}{\Phi} \cdot \operatorname{tg}^{2}\left(\frac{1}{2} k \sqrt{\Lambda \Phi} \cdot t+C_{1}\right)
$$

where $\Phi=3(1+w)+a_{1} \cdot k^{2}, C_{1}$ is an integration constant.

Then we obtain the gravitational equation for the dark matter:

$$
\dot{\rho}_{m}+\sqrt{3} k(1+w) \sqrt{\frac{3 \Lambda}{\Phi}} \operatorname{tg}\left(\frac{1}{2} \sqrt{\Lambda \Phi} \cdot t+C_{1}\right) \rho_{m}=Q
$$

We choose the interaction term between a dark energy and a dark matter in the following form:

$$
Q=\sin \left(\frac{1}{2} k \sqrt{\Lambda \Phi} \cdot t+C_{1}\right)
$$
by:

In this case the energy density of a dark matter is given

$$
\begin{gathered}
\rho_{m}=C_{2} \cdot\left[\cos \left(\frac{1}{2} k \sqrt{\Lambda \Phi} \cdot t+C_{1}\right)\right]^{\frac{6(1+w)}{\Phi}}+ \\
\sqrt{\frac{\Phi}{\Lambda}} \cdot \frac{\cos \left(\frac{1}{2} k \sqrt{\Lambda \Phi} \cdot t+C_{1}\right)}{3 k(1+w)\left[1-\frac{1}{2}\left(1-\frac{a_{1} k^{2}}{3(1+w)}\right)\right]},
\end{gathered}
$$

where $C_{2}$ is an integration constant.

Hubble's rate becomes:

$$
H(t)=k \sqrt{\frac{\Lambda}{\Phi}} \cdot \operatorname{tg}\left(\frac{1}{2} \sqrt{\Lambda \Phi} \cdot t+C_{1}\right)
$$

It occurs a periodic universe with Big Rip type singularity when

$$
t=t_{s}=\frac{\pi(1+2 n)-C_{1}}{k \sqrt{\Lambda \Phi}}, n \in Z .
$$

The derivative of $H(t)$ is equal to:

$$
\dot{H}(t)=\frac{k^{2} \Lambda}{2 \cos ^{2}\left(\frac{1}{2} \sqrt{\Lambda \Phi} \cdot t+C_{1}\right)}
$$

Thus, the universe expands.

The scale factor is given by the expression:

$$
a(t)=\exp \left[\int H(t) d t\right]=\frac{C_{2}}{\left[\cos ^{2}\left(\frac{1}{2} \sqrt{\Lambda \Phi} \cdot t+C_{1}\right)\right]^{\frac{1}{\Phi}}}
$$

The first and second derivatives of the scale factor are correspondingly given by:

$$
\begin{aligned}
& \dot{a}(t)=\frac{C_{2} \cdot \sin \left(k \sqrt{\Lambda \Phi} \cdot t+C_{1}\right)}{\left[\cos ^{2}\left(\frac{1}{2} k \sqrt{\Lambda \Phi} \cdot t+C_{1}\right)\right]^{\frac{\Phi^{2}-\Phi+2}{\Phi^{2}}}}, \\
& \ddot{a}(t)=k \sqrt{\Lambda \Phi} \cdot \dot{a}(t) \cdot\left[\operatorname{ctg}\left(k \sqrt{\Lambda \Phi} \cdot t+C_{1}\right)\right] . \\
& +\frac{\Phi^{2}-\Phi+2}{\Phi^{2}} \cdot \operatorname{tg}\left(\frac{1}{2} k \sqrt{\Lambda \Phi} \cdot t+C_{1}\right)
\end{aligned}
$$

If $0<t<\frac{\pi}{2 k \sqrt{\Lambda \Phi}}$, both derivatives are positive, the universe expands with acceleration (quintessence), and if $\frac{\pi}{2 k \sqrt{\Lambda \Phi}}<t<\frac{\pi}{k \sqrt{\Lambda \Phi}}$, one gets $\dot{a}(t)>0$ but $\ddot{a}(t)<0$, the universe expands with deceleration.

Let us suppose that both parameters $w_{1}, w_{2}$ and $\Lambda$ depend linearly on time:

$$
\begin{aligned}
& w_{1}(t)=w_{2}(t)=c t+b, \\
& \Lambda(t)=d t+e
\end{aligned}
$$

where $c, b, d, e$ are some constants. This kind of behaviour may be a consequence of the modification of the gravity (for a review, see [2]).

Then the equation (4) acquires the following form:

$$
\dot{\bar{\rho}}+\sqrt{3} k(c t+\theta) \bar{\rho}^{\frac{3}{2}}+\sqrt{3} k(d t+e) \bar{\rho}^{\frac{1}{2}}=0,
$$

where $\theta=b+\frac{\sqrt{3}}{3} a_{1} k^{2}+1$.

We shall investigate here, for further simplicity, the case $c e=d \theta$. The solution of the equation (15) looks like:

$$
\bar{\rho}=\frac{e}{\theta} \operatorname{tg}^{2}\left[\delta\left(t+\frac{\theta}{c}\right)^{2}+C_{1}\right]
$$

where $\delta=\frac{\sqrt{3 c d}}{4}, C_{1}$ is an arbitrary constant.

Let's write down the gravitational equation for the dark matter:

$$
\dot{\rho}_{m}+\sqrt{3} k(c t+b+1) \sqrt{\frac{e}{\theta}} \operatorname{tg}\left[\delta\left(t+\frac{\theta}{c}\right)^{2}+C_{1}\right] \rho_{m}=Q
$$


If we choose the interaction term in the form:

$$
Q=2 \delta\left(t+\frac{\theta}{c}\right) \cdot \sin \left[\delta\left(t+\frac{\theta}{c}\right)^{2}+C_{1}\right]
$$

then one obtains the solution of the equation (18) as:

$$
\rho_{m}=C_{2} \cos ^{2}\left[\delta\left(t+\frac{\theta}{c}\right)^{2}+C_{1}\right]-\cos \left[\delta\left(t+\frac{\theta}{c}\right)^{2}+C_{1}\right]
$$

Huble's rate is equal to:

$$
H(t)=\frac{k}{\sqrt{3}} \sqrt{\frac{e}{\theta}} \operatorname{tg}\left[\delta\left(t+\frac{\theta}{c}\right)^{2}+C_{1}\right]
$$

The periodic universe with finite-time cosmological singularity at $t=t_{s}= \pm \sqrt{\frac{\pi(1+2 n)-C_{1}}{2 \delta}}-\frac{\theta}{c}, n \in Z$ occurs. In this case, the energy-density and Hubble parameter simultaneously approach the infinity.

The time derivative of $H(t)$ becomes:

$$
\dot{H}(t)=\frac{k^{2}}{2} \sqrt{d} \cdot \frac{t+\frac{\theta}{c}}{\cos ^{2}\left(\delta\left(t+\frac{\theta}{c}\right)^{2}+C_{1}\right)}
$$

If $t>-\frac{\theta}{c}$, then the derivative is positive and the universe is expanding.

The scale factor is given by the expression:

$$
\begin{aligned}
& a(t)=\exp \left[\int H(t) d t\right]=a_{0} \cdot \exp \\
& {\left[\frac{\mu\left(S\left[\sqrt{\delta}\left(t+\frac{\theta}{c}\right)+C_{1}\right]-\frac{1}{2}\right)}{\cos ^{2}\left(\delta\left(t+\frac{\theta}{c}\right)^{2}+C_{1}\right)}\right]}
\end{aligned}
$$

where $\mu=\sqrt{\frac{2}{3} \pi \frac{e k}{\theta \sqrt{3 c d}}}, S\left[\sqrt{\delta}\left(t+\frac{\theta}{c}\right)\right]$ is Frenel's integral, $a_{0}$ is some constant.

$$
\text { If } t \rightarrow+\infty \text {, then } S\left[\sqrt{\delta}\left(t+\frac{\theta}{c}\right)\right] \rightarrow \frac{1}{2}, a(t) \rightarrow a_{0} .
$$

Hence, in this case the scale factor tends to constant and the universe tends to become the Minkowski space. The expansion is stopped, and the universe becomes static.

The first and second derivatives of the scale factor are correspondingly equal to:

$$
\begin{aligned}
& \dot{a}(t)=a(t) \cdot \mu \cdot \operatorname{tg}\left[\delta\left(t+\frac{\theta}{c}\right)^{2}\right] . \\
& \left\{1+2 \delta\left(t+\frac{\theta}{c}\right) \frac{\left(S\left[\sqrt{\delta}\left(t+\frac{\theta}{c}\right)\right]-\frac{1}{2}\right)}{\cos \left(\delta\left(t+\frac{\theta}{c}\right)^{2}+C_{1}\right)}\right\} \\
& \ddot{a}(t)=\dot{a}(t) \cdot\left[H(t)+\frac{4 \delta\left(t+\frac{\theta}{c}\right)}{\sin 2 \delta\left(t+\frac{\theta}{c}\right)^{2}}\right]+ \\
& 2 \mu \delta a(t) \cdot \operatorname{tg} \delta\left(t+\frac{\theta}{c}\right)^{2}
\end{aligned}
$$

$$
\left[\begin{array}{c}
{\left[\frac{1+2 \delta\left(t+\frac{\theta}{c}\right)^{2} \cdot \operatorname{tg} \delta\left(t+\frac{\theta}{c}\right)^{2}}{\cos \delta\left(t+\frac{\theta}{c}\right)^{2}}\right.} \\
\left.\left[S\left[\sqrt{\delta}\left(t+\frac{\theta}{c}\right)\right]-\frac{1}{2}\right)+\left(t+\frac{\theta}{c}\right) \operatorname{tg} \delta\left(t+\frac{\theta}{c}\right)^{2}\right]
\end{array}\right]
$$
tive, the universe expands with acceleration (quintessence). Thus, we constructed the oscillating universe which may appear after the matter-dominated phase [16] before acceleration.

\section{SUMMARY}

In this work we have studied a model of the coupling of a dark energy with a dark matter of special form in which we consider the account of the influence of the interaction between a dark energy and a dark matter on the evolution of the universe. The stationary and non-stationary cases for the parameters $w$ and $\Lambda$ in the equations of state are considered. Unlike a model with a pure dark energy, the presence of the interaction term between a dark energy and a dark matter in the special form in the equations of state, leads to a periodic appearance of Big Rip type singularity. It is possible in the non-stationary case that the universe tends to become the Minkowski space and becomes static in the remote future.

\section{ACKNOWLEDGEMENTS}

We thank professor Sergey Odintsov for very useful discussions and valuable remarks. 
The work was supported by the project, LRSS № 4489.2006.02 (Russia).

\section{REFERENCES}

[1] Nojiri S, Odintsov SD, Tsujikava. Properties of singularities in (phantom) dark energy universe. Phys Rev 2005; D71:063004, [hep-th/0501025].

[2] Nojiri S, Odintsov SD. Introduction to modified gravity and gravitational alternative for dark energy. Int J Geom Meth Mod Phys 2007; 4:115-146; Nojiri S, Odintsov S. D. Modified gravity with negative and positive powers of the curvature: unification of the inflation and of the cosmic acceleration. Phys Rev 2003; D68:123512, [hep-th/0307288].

[3] Nojiri S, Odintsov SD. Inhomogeneous Equation of State of the Universe: Phantom Era, Future Singularity and Crossing the Phantom Barrier. Phys Rev 2005; D72:023003, [hep-th/ 0505215]. Nojiri S, Odintsov SD. The new form of the equation of state for dark energy fluid and accelerating universe. Phys Lett 2006; B639: 144-150.

[4] Brevik I, Nojiri S, Odintsov SD, Vanzo L. Entropy and universality of Cardy-Verlinde formula in dark energy universe. Phys Rev 2004; D70:043520, [hep-th/ 0401073].

[5] Capozzielo S, Cardone V, Elizalde E, Nojiri S, Odintsov SD. Observational constraints on dark energy with generalized equations of state. Phys Rev 2006; D73:043512, [astro$\mathrm{ph} / 0508350]$.

[6] Brevik I, Gorbunova OG. Dark energy and viscous cosmology. Gen Rel Grav 2005; 37: 2039-2045.

[7] Nojiri S, Odintsov SD. The oscillating dark energy: future singularity and coincidence problem. Phys Lett 2006; B637: 139148.
[8] Brevik I, Gorbunova OG, Timoshkin AV. Dark energy fluid with

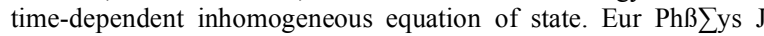
2007; C51: 179-183.

[9] Gorbunova OG. Ideal fluid and acceleration of the universe. Izw VUZov Fizika 2006; 49(5): 91-2.

[10] Ren J, Meng XH. Dark viscous fluid described by a unified equation of state in cosmology. Int J Mod Phys 2007; D16: 13411348; Ren J, Meng XH. Modified equation of state, scalar field and bulk viscosity in Friedmann universe. Phys Lett 2006; B636: 5-12; $\mathrm{Hu}$ M, Meng XH. Bulk viscous cosmology: Statefinder and entropy. Phys Lett 2006; B635:186-194; Cardone V, Tortora C, Troisi A, Capozziello S. Beyond the perfect fluid hypothesis for the dark energy equation of state. Phys Rev 2006; D73:043508, ar Xiv: 0511528 [astro-ph].

[11] B. McInnes. The dS / CFT correspondence and the big smash JHEP 2002; 0208:029, [hep-th/0112066].

[12] Nojiri S, Odintsov S. D. Quantum deSitter cosmology and phantom matter. Phys Lett 2003; B562: 147-52.

[13] Bamba K, Nojiri S, Odintsov SD. Future of the universe in modified gravitational theories: approaching to the finite-time future singularity. JCAP 0810:045 2008, arXiv: 0807.2575 [hepth].

[14] Nojiri S, Odintsov SD. Multiple Lambda cosmology: dark fluid with time-dependent equation of state as classical analog of cosmological landscape. Phys Lett 2007; B649: 440-444.

[15] Corda C. An oscillating Universe from the linearized $R^{\wedge}\{2\}$ theory of gravity. CRG 2008; 40: 2201, ar Xiv: 0802.2523 [astro-ph].

[16] Nojiri S, Odintsov SD, Stefancic H. On the way from matterdominated era to dark energy universe. Phys Rev 2006; D74:086009, [hep-th/0608168]. Elizalde E, Nojiri S, Odintsov S. D. Late-time cosmology in (phantom) scalar-tensor theory: dark energy and the cosmic speed-up. Phys Rev 2004; D70:043539, [hep-th/0405034].

(C) A.V. Timoshkin; Licensee Bentham Open.

This is an open access article licensed under the terms of the Creative Commons Attribution Non-Commercial License (http://creativecommons.org/licenses/by-nc/3.0/) which permits unrestricted, non-commercial use, distribution and reproduction in any medium, provided the work is properly cited. 